\title{
Screening for language development of preterm infants: relationship between two assessment instruments
}

Beatriz Servilha Brocchi ${ }^{1}$ https://orcid.org/0000-0002-9757-5382

Maria Cecília Marconi Pinheiro Lima² https://orcid.org/0000-0002-4203-0019

Pontifícia Universidade Católica de Campinas - PUC-Campinas, Campinas, São Paulo, Brasil.

Universidade Estadual de Campinas UNICAMP, Campinas, São Paulo, Brasil.

Conflict of interests: Nonexistent

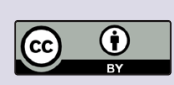

Accept on: August 26, 2021

Received on: May 20, 2021

Corresponding address:

Beatriz Servilha Brocchi

Avenida Jonh Boyd Dunlop, S/N,

Jd Ipaussurama

CEP:13060-904 - Campinas, SP, Brasil

E-mail: beatriz.servilha@puc-campinas. edu.br

\section{ABSTRACT}

Purpose: to associate two screening instruments used to assess global and language development of preterm infants.

Methods: a study including 69 children aged 0-24 months of corrected age, premature at birth, who were admitted to the Neonatal Intensive Care Unit (NICU). Researchers used an anamnesis and two protocols: Early Language Milestone Scale (ELM) and Observation and Monitoring Guide for Children from 0-48 months of age. According to the categorical and numerical variables, the protocols were associated with each other through descriptive percentages and non-parametric statistical tests, such as the Chisquare, Spearman's rank correlation coefficient and Mann-Whitney U, Kruskal-Wallis test and multiple correspondences $(p<0.05)$.

Results: a significant relationship was found between the Guide and the ELM, since the "not expected for the age" classification was associated with delayed/pathological classification in the Guide, while "expected" results were related to "adequate". There was also a relationship between the percentile of both instruments, as the higher the Guide value, the higher the ELM values (ELM-R $=0.564 ; p=0.000)$, suggesting that both protocols complement each other in the evaluation results.

Conclusion: the protocols for the assessment of language acquisition in children showed to be complementary, providing a complete assessment and relevant data on the development of preterm infants.

Keywords: Infant, Premature; Child Development; Language Development 


\section{INTRODUCTION}

Defined as the gestational age below 37 weeks, prematurity is understood as one of the main aggravating factors of neonatal mortality and morbidity, which results in negative impacts on Public Health ${ }^{1}$. The lower the gestational age, the greater the risk for mortality, abandonment and health problems, due to incomplete fetal development, thus, preterm children are deprived of a critical period of intrauterine growth ${ }^{2,3}$. In addition, premature birth also requires hospitalization, and a long stay in the Neonatal Intensive Care Unit (NICU) is often inevitable 4 .

The literature also describes disorders associated with preterm birth, which is reported as a biological risk factor for typical child development, increasing the likelihood of problems in different areas and moments of development, such as the delay in language acquisition in preterm children ${ }^{5,6}$.

It seems that the lower the birth weight and gestational age, the greater the probability of delays in the various stages of language development, such as: pre-linguistic milestones (e.g., recognizing objects, following verbal commands etc.), lower vocabulary and ability to form sentences at 2-3 years ${ }^{6}$.

Despite the frequency and extent of the verbal behavior of preterm babies being lower in relation to the normality pattern, the study found an approximation to this pattern of the infant born at term around 24 months. Given the high biological risks for development in the characterization of the language of preterm newborns, environmental factors and economic conditions are widely considered as aspects that affect the development process ${ }^{7,8}$.

In this context, the study by Isotami et al. (2009) which compared the expressive language of preterm and term children at two years of age, corroborates the information described above. Therefore, premature children had significantly lower expressive vocabulary than term children of the same age in all semantic categories. In addition, family income showed a positive association with phrasal length, as well as gestational age, birth conditions and birth weight.

Likewise, language alterations presented by preterm infants can be an impact resulting from the alteration in auditory maturity. In studies with term and preterm children, Rechia et al. (2016) ${ }^{9}$ and Azevedo \& Vieira $(1995)^{10}$, reported that early birth and the complications arising from this process may have impacts on auditory maturity, causing development to fall short of expected. Gouveia et al. (2020) ${ }^{11}$ also investigated the relationship between the auditory and language development of children aged between 18 and 36 months, comparing term and premature children at birth and reported changes in the auditory maturity of preterm children, as well as an association between the immaturity of this group with language alterations, thus reinforcing the presence of alterations in the auditory development in this population.

Given the difficulties observed in the development of preterm infants, this population requires a differentiated monitoring of their development and growth, so that possible changes can be identified early and early interventions can be implemented to minimize potential sequelae ${ }^{2,12,13}$, helping children to reach their maximum development, to integrate into their family and school environment and to achieve a good quality of life.

In this context, Law No. 13,257, of March 8, 2016, provides guidelines and directives for early childhood care, observing the importance of the first years of life for child development, and addressing child development monitoring (which encompasses from the gestational period to the 3-year-old child), as well as guidance to parents or guardians ${ }^{14}$. This law also provides for proper monitoring for all in early childhood.

The Joint Committee of Infant Hearing $(\mathrm{JClH})$, which is an international committee on childhood hearing, describes some peri-, pre- or postnatal complications as risk factors for hearing loss, including prematurity as one of the risk indicators for hearing impairment. Other risk factors may also occur together with prematurity, such as ICU stay for more than five days, or the occurrence of any of the following conditions, regardless of the length of stay in the ICU: need for extracorporeal ventilation; assisted ventilation; exposure to ototoxic drugs, such as aminoglycoside antibiotics and/or loop diuretics; hyperbilirubinemia; severe perinatal anoxia; Neonatal Apgar score from 0-4 in the first minute, or $0-6$ in the fifth minute; and birth weight less than 1,500 grams ${ }^{15}$.

Thus, the $\mathrm{JCIH}$ and the Multiprofessional Committee on Auditory Health (COMUSA), which is composed of Brazilian professionals who work in the area of hearing, especially children, recommend that all children undergo hearing assessment as soon as they are born and that those with risk factors for hearing loss receive hearing and language monitoring up to 3 years of age ${ }^{15,16}$. In addition, the Guidelines for Neonatal Hearing Screening, which were prepared by the Ministry of Health (2012), also recommend this monitoring, correcting the age of preterm children at birth. Thus, 
if any changes are detected during this follow-up, the child will be immediately referred to medical services and auditory rehabilitation in specialized services.

Although the interest of researchers and clinicians in language development has grown in recent years, there are still few standardized and validated instruments, especially to assess language development before 4 years of age ${ }^{17,18}$, and thus, this study used two protocols whose assessment starts with less than one month of life:

The ELM Scale (Early Language Milestone Scale), which was published in $1983^{19}$ and which in Brazil ${ }^{20}$ is called the "Escala de Aquisições Iniciais de Linguagem", evaluating infants with normal hearing and deaf infants. The ELM Scale has already been used as an instrument in many studies with children at high risk for deafness, with publications in many Scientific Journals in the field.

In addition to this scale, the study used the Observation and Monitoring Guide for Children from 0 to 48 months of age, which was developed ${ }^{21}$ in 1999 based on the expansion and updating of the Assessment Guide for the Development of Children from 0 to 36 months of age, which was prepared by the same authors in 1992. This guide understands "Language as a continuous process, which defines the human mind, whose success can be followed through behaviors"22. Through the observation of aspects of the child's global development, the guide aims to identify early changes in the processes of language acquisition and development, contemplating both verbal (language production and reception) and non-verbal aspects in the development process of the child. The evaluated aspects are organized into 3 levels: motor, cognitivesocial and auditory-verbal (including language), distributed by quarterly age groups between 0 and 18 months and semiannually between 19 and 48 months.

Both instruments are easy to apply and, when combined, cover areas that directly influence the development of children's language skills.

Thus, preterm newborns have a biological risk for language development alterations and the early detection of these alterations, through formal instruments, may help to mitigate the risks and enhance the development of preterm infants. Therefore, this study aimed to relate two screening instruments to assess the global and language development of preterm infants.

\section{METHODS}

\section{Participants}

This is a prospective cross-sectional quantitative study investigating the language development of children aged 0 to 24 months with a diagnosis of prematurity at birth. This study was approved by the Research Ethics Committee (CEP under the no. 2.326.785/2017) of the Pontifícia Universidade Católica de Campinas, Brazil, and is in accordance with Resolution No. 466, of December 12, 2012.

The study included 69 children aged 0 to 24 months of corrected age with a diagnosis of prematurity at birth, who were born and were admitted to the NICU of a teaching hospital in a city in the state of São Paulo. All participants were monitored at the High Risk Outpatient Clinic of this hospital from October 2017 to November 2018.

This service is characterized by longitudinal follow-up (up to 4 years of age) of children who were admitted to the NICU, born with less than 30 weeks of gestational age and/or with birth weight equal to or less than $1500 \mathrm{~g}$. In addition, infants with neurological disorders or syndromes (in this case, premature or at term) are also monitored. There is a multidisciplinary follow-up that includes pediatricians, nurses, speechlanguage pathologists, physiotherapists, occupational therapists and nutritionists, which is carried out monthly until the $6^{\text {th }}$ month of life; and every 3 months, up to one year of age. Then, the assessments become quadrennial after the $1^{\text {st }}$ year of life and up to 2 years; and annual after that age.

Since it is one of the criteria established by the service (all children are evaluated by the multidisciplinary team based on this), this study used the corrected age to assess the participants. Furthermore, the use of corrected age in the assessment of growth and development up to 2 years of age shows a real expectation for each child, without underestimating the preterm when comparing it with reference standards.

The study included all children within the defined age group, diagnosed with prematurity at birth (with no presence of neurological, auditory, visual malformations or syndrome alterations in the clinical evaluation performed by the pediatricians), whose parents signed the Informed Consent Form and who performed the entire proposed evaluation.

In turn, the study excluded participants who were younger or older than the proposal, and/or who had neurological, auditory, visual or syndrome disorders 
and who did not complete the assessment. Due to the above criteria, 6 children were excluded due to incomplete assessment and, therefore, the study was carried out with 69 children.

The participating children had a mean corrected age of 5.7 months $(S D=6.6)$, with a minimum age of 0 months (in which case participants would be considered newborns at the time of assessment) and a maximum age of 23 months. Most participants $(71 \%)$ were aged between 0-6 months, followed by the number of participants between 13-20 months (14.49\%), 7-12 months (8.6\%) and $14-23$ months (5.8\%).

\section{Materials}

The following instruments were used in this study:

- Anamnesis guide 23 : This guide has questions regarding the identification data of the child (age and date of birth), parents (age, date of birth, education and work), address, telephone number and monthly income. In addition, there are questions regarding previous maternal history, pregnancy planning, complications in the gestational process, medications used, use of legal and illegal drugs and maternal diseases. The instrument also collected the child's hospitalization history, such as gestational age, weight, length of stay, occurrences during the stay in the NICU and exams performed. Finally, the participants were asked about the entire hospitalization period, regarding the maternal-infant interaction (how the mother felt when she learned that the baby would be premature, and feelings during hospitalization) and the mother's daily activities with the child.

- Early Language Milestone Scale24: This scale is used as a screening tool for the language development of children aged 0 to 36 months, helping to determine patterns of linguistic behavior expected for each stage of child development. The instrument is easy to perform and is performed directly with the child or as a questionnaire for parents including 41 items in total, divided into three skills: auditory-expressive, auditory-receptive and visual skills (these skills combined make up the global score and the percentile).

a. Auditory expressive function: E1 (chirp); E2 (reciprocal vocalization); E3 (laugh); E4 (bubbles); E5 (monosyllabic babble); E6 (monosyllabic babble); E7 (mommy/daddy for anyone); E8 (mommy/daddy correctly);
E9 (first word other than mommy/daddy); E10 (production of 4 to 6 unique words).

b. Auditory receptive function: R1 (voice alert); R2 (lateral voice orientation); R3 (recognizes sounds); R4 (side bell); R5 (vertical bell); R6 (inhibits to a "no"); R7 (diagonal bell-location direct down and indirect up); R8 (command with no gesture); R9 (points to more than one part of the body); R10 (two commands with no gestures).

c. Visual function: V1 (smiles); V2 (recognizes parents); V3 (recognizes objects); V4 (responds to facial expressions); V5 (visual-horizontal and vertical tracking); V6 (blinks to danger); V7 (imitates gestural games); V8 (command with gesture); V9 (imitates gestural games); V10 (points to objects).

Each skill is represented by its description (such as asking for something, recognizing parents, and recognizing objects) and by a rectangle that is divided into 4 parts. Each part is equal to the percentage of children who took the test. Through questioning the parents and observing the behavior by the evaluator, it was possible to assess whether the item expected for the age was already performed by the child. In this way, the items performed by the participants were marked, while an "X" was noted on items that the participant did not do yet.

Observation and Monitoring Guide for Children from 0 to 48 months of age ${ }^{21}$ : This instrument aims to identify early changes in language acquisition and development processes, within the development monitoring routine. The instrument assesses verbal (language emission and reception) and non-verbal manifestations in the child's evolution process.

Thus, the evaluator must note whether or not the child performed a certain behavior in an appropriate column. All notes and analyzes were performed in the protocol itself. The instrument also has questions related to the identification of the subject, physical and behavioral description based on the date and chronological age, and observations of the child's behavior. Thus, the Guide allows for the classification of development as adequate, at risk, delayed and pathological.

The results were then transcribed into specific protocols and the analysis was performed by the author, a speech-language pathologist, who is a specialist in child language and trained to use the instruments described above. 


\section{Procedures}

In the routine of pediatric assessment at the High Risk Outpatient Clinic, the evaluator and the medical team verified which patients who were scheduled for the day were within the inclusion criteria of the study. Then, the family was welcomed by the evaluator who introduced the project and requested the signing of the Informed Consent Form. This should be done in the waiting room, before the child's consultation with pediatrics and the multidisciplinary team.

The questions related to the anamnesis were asked after this procedure (preceding the multidisciplinary follow-up), and then the corrected age was calculated with the date of birth and gestational age. Then, the ELM scale was applied from this calculation by asking the parents. To this end, a line was drawn with the child's age and then the parents were asked about the items performed by the children. In addition, if the child was awake, the observation of the child's behavior would complement these data. When parents reported that the child already performed an item (such as visually following an object), the evaluator should understand it as a present behavior; on the other hand, if the parents reported that the child was not able to do perform an item, it was understood as an absent behavior. At the end, the parents were instructed on language development and ways to stimulate the child in their daily routine.

\section{Data analysis}

The protocols were related to each other based on categorical and numerical variables for each instrument.

- ELM Scale: The evaluator drew a line at the child's corrected age and verified, through the answers collected with the parents, the skills and percentage of items performed by the evaluated child. Each skill was given a score and, based on the child's age, the evaluator verified if the child was within the expected limits for the age (by skill), according to a specific score table. The scores for all aspects observed were also added up to obtain a total score that was also compared to the table to check the child's performance across the instrument. Thus, it was possible to obtain a numerical variable (value of each aspect and the global value) and a categorical variable (expected/unexpected for the age in each aspect and in the global score).

- Observation and Development Guide: the Guide consists of expected behaviors for an age group (divided quarterly) into 3 categories: the 'motor aspects' category has 10 items; while the 'cognitive-social' has 4 items and the 'auditory-verbal' has 5 items for all ages (all 10 items described are distributed in the same proportion by age range assessed). After summing up the results obtained in an interview with the parents, the evaluators calculated a percentage of the participant's performance based on the total number of items (100\%), thus calculating the percentage of the child's total items within the corrected age group. This percentile was compared to the performance table that was developed by the authors of the instrument and, as so, the participant could be classified as pathological, delayed, at risk or adequate. Participants classified as "delayed" and "pathological" were combined to obtain the number of participants needed to apply the statistical tests for the analysis of the studied population.

To this end, the researchers used descriptive percentages and non-parametric statistical tests, such as Chi-square test, Spearman's rank correlation coefficient and Mann-Whitney $\mathrm{U}$ test, Kruskal-Wallis test, and multiple correspondences, which were performed using the SPSS 2.0 software. The presence of correlation and alternative hypothesis $\mathrm{H} \neq 0$ was adopted as the basic hypothesis $\left(\mathrm{H}_{0}=0\right)$, for a 0.05 significance level, which is suitable for human sciences.

\section{RESULTS}

Regarding the birth conditions of the participants, as shown in Table 1, almost half (49.2\%) were found to be extreme preterm, as they were born between 24 and 30 weeks of gestational age (GA). Table 1 also shows that the mean $\mathrm{GA}$ was 30.93 weeks $(\mathrm{SD}=2.4)$.

The study also had thirty-two children $(46.3 \%)$ who were moderately premature ( 31 to 34 weeks of gestational age) and only one (1.44\%) was considered late premature (36 weeks of gestational age at birth).

Birth weight was on average 1,427.14 g $(S D=551.24)$. In addition, half of the participants (55.1\%) weighed $1001-1500 \mathrm{~g}$ at birth, followed by participants weighing over $1500 \mathrm{~g}(27.5 \%)$ at birth and $17.4 \%$ who were born weighing less than $1000 \mathrm{~g}$.

The participants had an APGAR score at 1 minute of $6.3(\mathrm{SD}=2.8)$, and an APGAR score at 5 minutes of $8.7(\mathrm{SD}=1.2)$. The children's hospital stay was on average 50.96 days $(S D=23.3)$, with a minimum of 8 and a maximum of 90 days. 
Table 1. Birth conditions of study participants

\begin{tabular}{lccccc}
\hline & $\begin{array}{c}\text { Apgar score at } \\
\text { 1 minute }\end{array}$ & $\begin{array}{c}\text { Apgar score at } \\
\mathbf{5} \text { minutes }\end{array}$ & Weight & GA & $\begin{array}{c}\text { Length of stay } \\
\text { (days) }\end{array}$ \\
\hline Mean & 6.30 & 8.70 & $1,427.14$ & 30.93 & 50.96 \\
Median & 8.00 & 9.00 & $1,262.50$ & 31.00 & 47.00 \\
Minimum & 1 & 5 & 850 & 27 & 8 \\
Maximum & 9 & 10 & 3,155 & 36 & 90 \\
Standard deviation & 2.826 & 1.235 & 551.248 & 2.433 & 23.381 \\
\hline
\end{tabular}

Caption: $\mathrm{GA}=$ gestational age

Regarding the Early Language Milestone Scale (ELM), the researchers observed children's performance by category (auditory-expressive, auditoryreceptive and visual) and as a whole, through the global performance.

As shown in the table above, the participants recorded an average performance of $5.3(S D=4.8)$ in the auditory-expressive category, which is equivalent to the performance of children aged 7.2 months, according to the author of the test. It should be noted that more than half of children (64.3\%) performed within expectations for the corrected age.
Similarly, the performance of children in the auditoryreceptive category had an average of $4.6(\mathrm{SD}=4.1)$. Half of the participants performed as expected for the corrected age, which was equivalent to the age of 6.2 months. In turn, the visual category had the highest average $(5.5 ; \mathrm{SD}=2.9)$ and more than half of the children $(60.7 \%)$ were within the expected range for their age.

As for the overall performance of the subjects (all the 3 categories combined), the children had an average of $15.4(\mathrm{SD}=6.3)$, with an age equivalent to 6.3 months. It should be noted that just over half of the children (57.1\%) performed as expected for the corrected age.

\section{ELM}

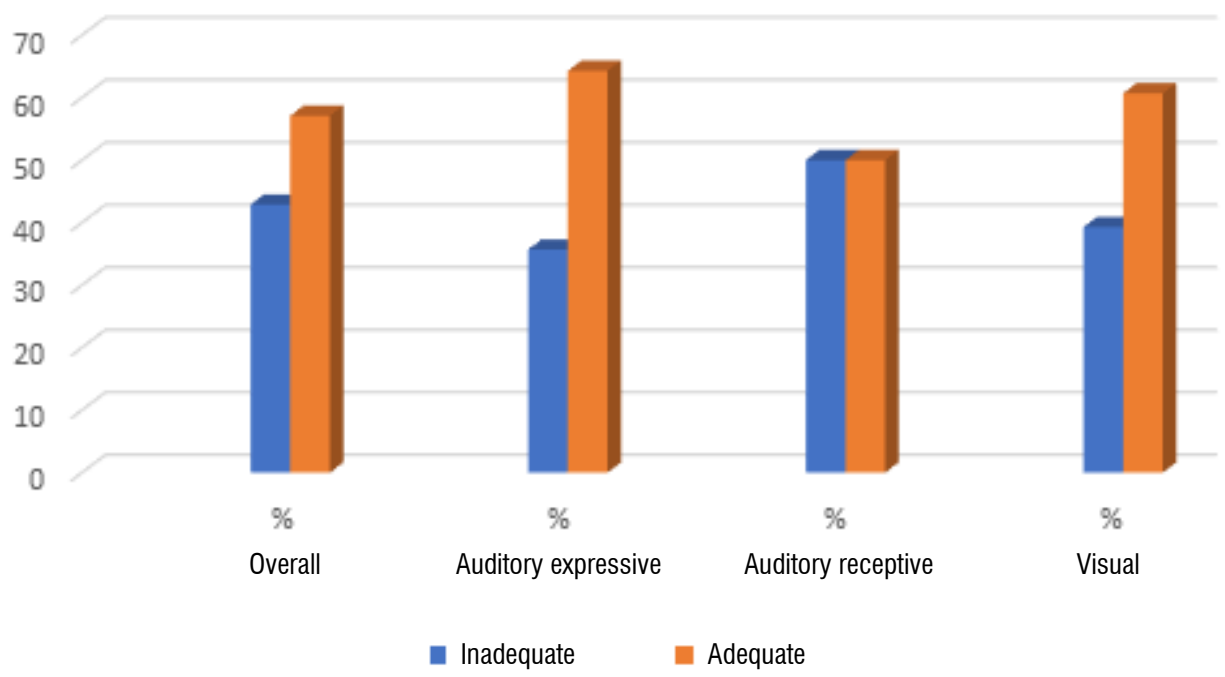

Caption: ELM: Early Language Milestone Scale

Figure 1. Expected performance for participants based on the Early Language Milestone Scale 


\section{Observation and Development Guide}

The average percentile found by the researchers among the participants was 81.2 (SD=16.2). In addition, more than half of the participating children showed developmental changes, as shown in Table 3. In turn, $21.4 \%$ of the participants were adequate and a quarter were at risk.

\section{Development Guide}

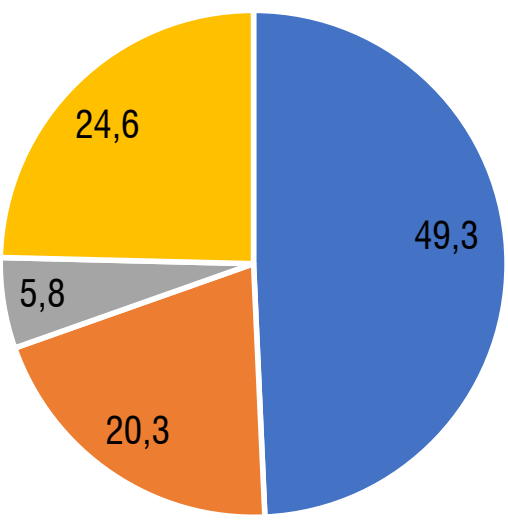

Adequate $\square$ Delayed $\square$ Pathological $\square$ At risk

Figure 2. Performance of participants in the Diagnosis and Follow-up Guide
A relationship between the percentile of both instruments was found from the statistical analysis performed between numerical variables of the ELM Scale (by skill and global score) and the Development Guide (through Spearman's rank correlation coefficient), which suggests that the higher the Guide value, the higher the ELM Scale values (ELM-R=0.564; $p<0.001$ ).

Then, after the analysis of numerical parameters, the categorical variables of the tests were considered. From the ELM Scale, the researchers used the global score and the percentile of "expected for their age" and "unexpected for their age" for each skill. In turn, the performance in the Development Guide was classified as adequate, at risk and delayed/pathological (with these two classifications being grouped for the performance of the statistical analysis). The researchers also decided to use the Chi-square test for comparison between groups.

As shown in Table 1, all aspects of the ELM Scale are related to the Developmental Guidance and, except for the auditory-expressive ability of the ELM Scale, a significant association with the Developmental Guidance rating was found in all other skills. In the ELM Scale, the "not expected for the age" classification was associated with delayed/pathological in the Guide, while the "expected" classification was related to adequate.

Table 2. Correlation between the categorical variables of the instruments (Early Language Milestone Scale and Guide)

\begin{tabular}{|c|c|c|c|c|c|}
\hline \multirow{2}{*}{ ELM } & & \multicolumn{3}{|c|}{ Guide } & \multirow{2}{*}{ Chi-square test } \\
\hline & & Appropriate & Delayed/pathological & Risk & \\
\hline \multirow{2}{*}{ Auditory expressive } & esperado & $45.00 \%$ & $22.50 \%$ & $32.50 \%$ & \multirow{2}{*}{$<0.001 *$} \\
\hline & não-esperado & $7.10 \%$ & $64.30 \%$ & $28.60 \%$ & \\
\hline \multirow{2}{*}{ Auditory receptive } & esperado & $51.40 \%$ & $17.10 \%$ & $31.40 \%$ & \multirow{4}{*}{$<0.001 *$} \\
\hline & não esperado & $5.30 \%$ & $63.20 \%$ & $31.60 \%$ & \\
\hline \multirow{2}{*}{ Visual } & esperado & $48.70 \%$ & $23.10 \%$ & $28.20 \%$ & \\
\hline & não-esperado & $0 \%$ & $60 \%$ & $40 \%$ & \\
\hline \multirow{2}{*}{ Overall } & esperado & $46.20 \%$ & $17.90 \%$ & $35.90 \%$ & \multirow{2}{*}{$<0.001 *$} \\
\hline & não-esperado & $6.70 \%$ & $73.30 \%$ & $20 \%$ & \\
\hline
\end{tabular}

*significance level at $p<0.05$

Caption: ELM: Early Language Milestone Scale 
In addition, other correlations of the numerical data of the ELM Scale were also performed from the findings of the previous analyses, for each skill, global score and percentile with the categorical findings of the Guide (adequate, at risk and delayed/pathological). The researchers decided to use the Kruskal-Wallis Test with Tukey's range test for multiple comparisons, when significant, for this type of analysis.
In this way, a significant relationship was found between the Guide and the percentile score $(p<0.001)$ of the ELM $(p<0.05)$. In turn, the 'Adequate' aspect had higher values in relation to Delayed/pathological (Table 3 ) in the multiple comparisons of these two aspects.

Table 3. Association between numerical variables from the Early Language Milestone Scale and categorical variables from the Observation Guide

\begin{tabular}{|c|c|c|c|c|c|c|}
\hline & \multicolumn{3}{|c|}{ Guide } & \multirow{2}{*}{$\begin{array}{c}\text { Kruskal-Wallis } \\
\text { Test (p) }\end{array}$} & \multirow{2}{*}{$\begin{array}{l}\text { 2x2 Multiple } \\
\text { Comparisons }\end{array}$} \\
\hline & & Appropriate & Delayed/pathological & Risk & & \\
\hline \multirow{3}{*}{$\begin{array}{l}\text { Auditory } \\
\text { expressive }\end{array}$} & Mean & 4.79 & 4.67 & 7.06 & \multirow{3}{*}{0.328} & \\
\hline & Median & 4.00 & 4.00 & 4.00 & & \\
\hline & Standard deviation & 4.44 & 3.51 & 5.58 & & \\
\hline \multirow{3}{*}{$\begin{array}{l}\text { Auditory } \\
\text { receptive }\end{array}$} & Mean & 4.53 & 4.11 & 5.94 & \multirow{3}{*}{0.202} & \\
\hline & Median & 3.00 & 2.50 & 5.00 & & \\
\hline & Standard deviation & 4.95 & 3.03 & 3.94 & & \\
\hline \multirow{4}{*}{ Visual } & Mean & 5.68 & 5.17 & 6.71 & \multirow{3}{*}{0.500} & \\
\hline & Median & 6.00 & 6.00 & 6.00 & & \\
\hline & Standard deviation & 2.00 & 3.00 & 3.04 & & \\
\hline & Mean & 15.00 & 13.94 & 19.71 & \multirow{3}{*}{0.324} & \\
\hline \multirow[t]{3}{*}{ Overall } & Median & 11.00 & 12.50 & 15.00 & & \\
\hline & Standard deviation & 10.92 & 9.00 & 12.10 & & \\
\hline & Mean & 84.37 & 40.06 & 62.71 & \multirow{3}{*}{$0.001^{*}$} & $(1 \times 2) p=0.001^{*}$ \\
\hline \multirow[t]{2}{*}{ Percentile } & Median & 90.00 & 40.00 & 75.00 & & $(1 \times 3) p=0.181$ \\
\hline & Standard deviation & 21.35 & 35.62 & 48.00 & & $(2 \times 3) p=0.163$ \\
\hline
\end{tabular}

*significance level at $p<0.05$.

Finally, and in order to fully investigate the relationship between the two instruments, the researches associated the categorical variables of the ELM ('expected for the age' and 'not expected for the age') with the categorical variables of the Guide (adequate, at risk and delayed/pathological). Thus, Table 4 shows a significant correlation in all aspects analyzed. As shown in the results, the "expected for the age" aspect was associated with the highest values of the percentile of the Observation and Monitoring Guide.

Table 4. Association between categorical variables from the Early Language Milestone Scale and numerical variables from the Observation Guide

\begin{tabular}{|c|c|c|c|c|c|}
\hline ELM & & Guide Mean & Median & Standard deviation & $\begin{array}{c}\text { Mann-Whitney U } \\
\text { test (p) }\end{array}$ \\
\hline \multirow[b]{2}{*}{ Auditory expressive } & Expected & 83.3 & 89.3 & 13.48 & \multirow{2}{*}{$0.001^{*}$} \\
\hline & Unexpected & 63.75 & 68.2 & 18.06 & \\
\hline \multirow{2}{*}{ Auditory receptive } & Expected & 85.42 & 89.4 & 12.15 & \multirow{2}{*}{$<0.001^{*}$} \\
\hline & Unexpected & 64.99 & 65 & 65 & \\
\hline \multirow{2}{*}{ Visual } & Expected & 83.18 & 89.4 & 13.93 & \multirow{2}{*}{$0.001^{*}$} \\
\hline & Unexpected & 65.37 & 68.4 & 17.88 & \\
\hline \multirow{2}{*}{ Overall } & Expected & 83.5 & 89.4 & 89.4 & \multirow{2}{*}{$<0.001^{*}$} \\
\hline & Unexpected & 64.54 & 65 & 14.13 & \\
\hline
\end{tabular}

*significance level at $p<0.05$; Caption: ELM: Early Language Milestone Scale 
Given the data described above, a significant relationship was found between the two instruments used in the study with preterm infants through different statistical tests.

\section{DISCUSSION}

There is a consensus in the literature on the relevance of early monitoring of prematurely born children ${ }^{2,6,13,20,25}$. In these cases, the biological conditions associated with environmental issues are risk factors for the development of these children ${ }^{2,6,7,13,25}$.

Since preterm infants are deprived of a critical period of intrauterine growth, which occurs in the last trimester of pregnancy ${ }^{25}$, medical complications are very common, such as septicemia, apnea, bradycardia and chronic lung diseases, among others, thus requiring intervention and a longer stay in the NICU. When added to biological immaturity, all these factors may have a significant impact on the child's brain development, as the child's central nervous system was not yet fully prepared to work outside the intrauterine environment ${ }^{26}$.

As neurobiological immaturity may lead to negative impacts on the development of preterm infants, the assessment of the global development of preterm infants is critical for the early detection of motor, behavioral, cognitive and language alterations. In this sense, Lamônica \& Picolini $(2009)^{6}$ investigated the performance of linguistic, cognitive, motor, self-care and socialization development skills in 30 preterm children of both genders, aged 6 to 24 months. In this study, the authors found that all children of the studied age group had linguistic and self-care deficits when compared to term children of the same age group. Another study ${ }^{27}$ on neurobehavioral assessment of preterm children found that they have more frequent alterations in the language, adaptive behavior, socio-emotional and eye-motor aspects. Considering the risks of the preterm population for their global development, this study used the Observation and Monitoring Guide for Children from 0 to 48 months of age.

It should be noted that language is the most frequent alteration in this population, according to the researched literature. Therefore, the ELM Scale was included in the assessment. Similar to the Guide, the ELM Scale is quick and easy to perform and evaluates the expressive and receptive language, as shown by a previous study ${ }^{20}$.

Oliveira, Lima \& Gonçalves (2003) ${ }^{20}$ carried out a longitudinal analysis of preterm and full-term infants and found that, although there was no statistically significant difference between the two groups, preterm children with low birth weight had a delay in the development of verbal expression in relation to children born at term. Furthermore, another longitudinal study that evaluated the language and cognition performance of very low birth weight preterm children found that expressive language developed late and that the delay persisted throughout the sensorimotor period until the preoperative period ${ }^{28}$.

It should be noted that language alterations in this population may be due to alterations in the maturation of the central auditory pathway. Since preterm birth is a risk factor for the maturation process of the central auditory system, harming the hearing of premature children, ${ }^{9,10}$ premature neonates with alterations in auditory development may have worse language performance ${ }^{29}$. A study with 66 children (term and preterm infants) investigated the relationship between the development of auditory skills in the first year of life and language development in preterm children with chronological age between 18 and 36 months ${ }^{11}$. In this context, the group with normal auditory development had better results in language assessment, especially in the receptive aspects.

The two instruments used together showed satisfactory agreement, which means that the 'adequate' classification of the Guide was associated with results 'within the expected' of the ELM scale, while the 'unexpected' was associated with 'delayed/pathological', showing the complementarity and synchrony of both tests together in the assessment of this population.

The use of the two instruments combined makes it possible to monitor global development and assess communication and language aspects in detail. The Guide has 5 objective questions by age group about auditory behavior (for example, if the child locates the sound directly down), verbal (if the child uses syllables) and non-verbal communication (if the child babbles), while the ELM complements the Guide by including more aspects regarding comprehension (for example, if the child understands "no"), expression (use of words, such as "daddy" and "mommy") and visual issues (e.g., if the child visually follows an object). Given the risk factors involved in preterm birth, the combined tests proved to be complementary, as they assessed aspects related to visual, auditory and language issues. According to the studies described above $e^{6,20,27}$, these aspects are risk areas for premature infants and allow 
for the early detection of delays or alterations for the purpose of early intervention.

In turn, the authors of a review of assessment instruments for preterm infants proposed that the examiner should choose the examination that best suits the objectives among the many tests used for screening and diagnosing developmental abnormalities in preterm infants, whether in their use in research, clinical screening or in verifying the efficiency of the proposed early intervention ${ }^{30}$. In addition, the authors recommend investigating the clinical conditions and age range to select an instrument.

In this study, all children were aged from 0 to 24 months, and even with age correction, all could be evaluated. Thus, given their easy application and effectiveness in monitoring global and language development, both tests can be used in childcare and development monitoring speech-language pathology services.

Programs such as the auditory monitoring of children with Risk Indicators for Hearing Loss may benefit from these protocols. The monitoring recommended by the Ministry of Health ${ }^{17}, \mathrm{JClH}^{15}$ and $\mathrm{COMUSA}^{16}$ highlights that, after performing neonatal hearing screening with satisfactory results, every child with a history of hearing loss in the family or pre, peri or postnatal complications, in addition to maternal complications during the pregnancy, should undergo childcare consultations in Primary Care to assess auditory and language development up to 3 years of age. In this program, the use of both protocols will provide the examiner with relevant data on the child's global and language development. According to the guidelines for Neonatal Hearing Screening ${ }^{17}$, the ages for preterm infants considered during monitoring refer to the corrected age, as used in this study.

Therefore, this study focused on the investigation of children premature at birth and recommends that further studies investigate the use of both protocols in other populations and age groups.

\section{CONCLUSION}

The results of this study show that the two protocols, the Observation and Monitoring Guide and the Early Language Milestone Scale (ELM), are complementary for the assessment of language acquisition in children, providing a complete assessment with relevant data on the development of preterm infants. Thus, the monitoring of language acquisition processes of premature children and timely intervention may help to prevent or reduce the sequelae resulting from premature birth.

\section{REFERENCES}

1. Organização Mundial de Saúde. Preterm birth. [Homepage on the Internet] [accessed on 2018 June 19]. Available at: http://www.who.int/ news-room/fact-sheets/detail/preterm-birth.

2. Ribeiro CC, Lamônica DAC. Communicative abilities in premature and extreme premature infants. Rev. CEFAC. 2014;16(3):830-9.

3. Teixeira LA, Vasconcelos LD, Ribeiro RAF. Prevalence of disease na relationship to preterm birth in high-risk pregnancy. Rev. Ciên.Saúd. 2015;5(4):35-2.

4. Melo MRO, Andrade ISNS. Child development and prematurity: a reflection on maternal knowledge and expectations. Rev Bras. Prom. Saúd. 2013;26(4):548-53.

5. Linhares MBM. Estresse, resiliência e cuidado no desenvolvimento de neonatos de alto risco. In: Mendes EG, Almeida MA, Williams LCA, editors. Temas em educação especial: avanços recentes. São Carlos: EDUFSCar, 2004. p. 315-24.

6. Lamônica DAC, Picolini MM. Habilidades do desenvolvimento de prematuros. Rev. CEFAC. 2009;11Supl(2):145-53.

7. Isotami SM, Azevedo MF, Chiari BM, Perissinoto J. Linguagem expressiva de crianças nascidas pré-termo e termo aos dois anos de idade. Pró-Fono R. Atual. Cientif. 2009;21(2):155-60.

8. Foster-Cohen S, Edgin JO, Champion PR, Woodward LJ. Early delayed language development in very preterm infants: evidence from the MacArthur-Bates CDI. JChild Lang. 2007;34(3):655-75.

9. Rechia IC, Oliveira LD, Crestani AH, Biaggio EP, Souza AP. Effects of prematurity on language acquisition and auditory maturation: a systematic review. CoDAS. 2016;28(6):843-54.

10. Azevedo M, Vieira R. Desenvolvimento auditivo de crianças normais e de alto risco. Plexus Editora; 1995.

11. Gouveia AS, Oliveira MMF, Goulart AL, de Azevedo MF, Perissinoto J. Development of speech and hearing skills in prematures adequate and small for gestational age: chronological age between 18 and 36 months. CoDAS. 2020;32(4):e20180275.

12. Guarini A, Marini A, Savini S, Alessandroni R, Faldella G, Sansavini A. Linguistic features in 
children born very preterm at preschool age. Dev. Med. Child Neurol. 2016;58(9):949-56.

13. Mello RR, Meio MDBB. Follow-up de recém-nascido de risco. In. Moreira MEL, Braga NA, Morsch DS, editors. Quando a vida começa diferente: o bebê a sua família na UTI neonatal. Rio de Janeiro. Editora Fiocruz; 2003. p.179-84.

14. Brasil, 2016 Presidência da República, Casa Civil Lei № 13.257, de 8 de Março de 2016. [Homepage on the Internet] [accessed on 2021 May 11]. Available at: http://www.planalto.gov.br/ccivil_03/_ ato2015-2018/2016/lei/l13257.htm

15. Joint Committee on Infant Hearing. Year 2019 position statement: principles and guidelines for early hearing detection and intervention programs. J Early hear det interv. 2019;4(2):1-44.

16. Lewis DR, Marone SAM, Mendes BCA, Cruz OLM, Nóbrega M. Comitê multiprofissional em saúde auditiva: COMUSA. Braz. J. Otorhinolaryngol. 2010;76(1):121-8.

17. Brasil. Ministério da Saúde. Secretaria de Atenção à Saúde. Departamento de Ações Programáticas Estratégicas. Diretrizes de Atenção da Triagem Auditiva Neonatal / Ministério da Saúde, Secretaria de Atenção à Saúde, Departamento de Ações Programáticas Estratégicas e Departamento de Atenção Especializada. - Brasília: Ministério da Saúde, 2012.

18. O'Neil D. The language use inventory for young children: a parent-report measure of pragmatic language development for 18 to 47-months-old children. J Speec, Lang Hear Res. 2007;50(1):214-28.

19. Coplan J. The Early Language Milestone Scale. Pro-ED, Austin, 1983. Revisada em 1993.

20. Oliveira LN, Lima MCMP, Gonçalves VMG. Acompanhamento de lactentes com baixo peso ao nascimento. Arq neuropsiq. 2003;61(3):802-7.

21. Perissinoto J, Pedromônico MRM, Azevedo MF, Silva AAM, Tavares FM, Isotami SM. Roteiro de observação e acompanhamento do desenvolvimento de crianças de 0 a 48 meses de idade. Anais de IV Congresso Internacional de Fonoaudiologia e III Encontro Ibero-americano de Fonoaudiologia. São Paulo. 1999:46.

22. Perissinoto J, Isotani SM. Desenvolvimento da linguagem: programa de acompanhamento de recém nascidos de risco. In: Hernandez AM, editor. Conhecimentos essenciais para atender bem $\mathrm{O}$ neonato. São José dos Campos: Pulso; 2003. p.113-21.

23. Brocchi BS, Leme MIS. Relation between motherchild interaction upon the development of oral language of the preterm newborn. Audiol. Commun Res. 2013;18(4):321-31.

24. Lima MCMP. Avaliação de fala em lactentes no período pré-linguístico: uma proposta para triagem de problemas auditivos [Dissertation]. Campinas (SP): Faculdade de Ciências Médicas, Universidade Estadual de Campinas; 1997.

25. Rugolo LMSS. Crescimento e desenvolvimento a longo prazo do prematuro extremo. J. Pediatr. 2005;81(1):101-10.

26. Carniel CZ, Furtado MCC, Vivente JB, Abreu RZ, Tarozzo RM, Cardia SETR et al. Influence of risk factors on language development and contributions of early stimulation: an integrative literature review. Rev. CEFAC. 2017;19(1):109-18.

27. Fernandes LV, Goulart AL, Santos AM, Barros MC, Guerra CC, Kopelman BI. Neurodevelopmental assessment of very low birth weight preterm infants at corrected age of 18-24 months by Bayley III scales. J Pediatr. 2012;88(6):471-8.

28. Buhler KE, Limongi SC, Diniz EM. Language and cognition in very low birth weight preterm infants with PELCDO application. Arq Neuropsiquiatr. 2009;67(2A):242-9.

29. Luiz CBL, Garcia MV, Perissinoto J, Goulart AL, Azevedo MF. Relation between auditory abilities in the first year of life and language diagnosis in pre-terms. Rev. CEFAC. 2017;18(6):1316-22.

30. Silva NDSH, Lamy FF, Gama MEA, Lamy ZC, Pinheiro AL, Silva DN. Instrumentos de avaliação do desenvolvimento infantil de recém-nascidos prematuros. J Hum Grow Dev. 2011;21(1):85-98. 